JOURNAL OF

FUNCTION SPACES AND APPLICATIONS

Volume 9, Number 1 (2011), 41-66
(C) 2011, Scientific Horizon http://www.jfsa.net

\title{
Dunkl-Sobolev spaces of exponential type and applications
}

\author{
Hatem Mejjaoli \\ Dedicated to Khalifa Trimèche \\ (Communicated by Björn Birnir)
}

2000 Mathematics Subject Classification. Primary 46F15. Secondary $46 \mathrm{~F} 12$.

Keywords and phrases. Dunkl operators; Dunkl-Sobolev spaces of exponential type; Pseudo differential-difference operator; Reproducing kernel.

\footnotetext{
Abstract. We study the Sobolev spaces of exponential type associated with the Dunkl operators. Some properties including completeness and imbedding theorem are proved. Next we introduce a classes of symbols of exponential type and the associated pseudo-differential-difference operators, which naturally act on the Dunkl-Sobolev spaces of exponential type. Finally using the theory of reproducing kernels some applications are given for these spaces.
}

\section{Introduction}

We consider the differential-difference operators $T_{j}, j=1,2, \cdots, d$, on $\mathbb{R}^{d}$ introduced by C.F. Dunkl in [3]. These operators are very important in pure mathematics and in Physics. They provide a useful tool in the study of special functions with root systems [2], [6]. Moreover the commutative algebra generated by these operators has been used in the study of certain exactly solvable models of quantum mechanics, namely the Calogero-Sutherland-Moser models, which deal with systems of identical particles in a one dimensional space. 
Dunkl has proved in [4] that there exists a unique isomorphism $V_{k}$ from the space of homogeneous polynomials $\mathcal{P}_{n}$ on $\mathbb{R}^{d}$ of degree $n$ onto itself satisfying the transmutation relations

$$
T_{j} V_{k}=V_{k} \frac{\partial}{\partial x_{j}}, \quad j=1,2, \cdots, d,
$$

and $V_{k}(1)=1$. This operator is called Dunkl intertwining operator. Next $K$. Trimèche has extended this operator to an isomorphism from $\mathcal{E}\left(\mathbb{R}^{d}\right)$ (the space of $C^{\infty}$-functions on $\mathbb{R}^{d}$ ) onto itself satisfying the relation (H) (see [18]). The operator $V_{k}$ possesses the integral representation

$$
V_{k}(f)(x)=\int_{\mathbb{R}^{d}} f(y) d \mu_{x}(y), \quad f \in \mathcal{E}\left(\mathbb{R}^{d}\right), x \in \mathbb{R}^{d}
$$

where $\mu_{x}$ is a probability measure on $\mathbb{R}^{d}$ with support in the closed ball $B(0,\|x\|)$ of center 0 and radius $\|x\|$ (see [14], [18]). In [18] K.Trimèche has studied the transposed operator ${ }^{t} V_{k}$ of the operator $V_{k}$. It has the integral representation

$$
{ }^{t} V_{k}(f)(y)=\int_{\mathbb{R}^{d}} f(x) d \nu_{y}(x), \quad y \in \mathbb{R}^{d} .
$$

where $\nu_{y}$ is a positive measure on $\mathbb{R}^{d}$ with support in the set $\{x \in$ $\left.\mathbb{R}^{d} /\|x\| \geq\|y\|\right\}$ and $f$ in $D\left(\mathbb{R}^{d}\right)$ (the space of $C^{\infty}$-functions on $\mathbb{R}^{d}$ with compact support). This operator is called Dual Dunkl intertwining operator. K. Trimèche has proved also in [18] that the operator ${ }^{t} V_{k}$ is an isomorphism from $D\left(\mathbb{R}^{d}\right)$ onto itself, satisfying the transmutation relations

$$
{ }^{t} V_{k}\left(T_{j} f\right)(y)={\frac{\partial}{\partial y_{j}}}^{t} V_{k}(f)(y), \quad y \in \mathbb{R}^{d}, j=1,2, \cdots, d .
$$

Using the operator $V_{k}$, C.F. Dunkl has defined in [4] the Dunkl kernel by $K(x,-i z)=V_{k}\left(e^{-i\langle\cdot, z\rangle}\right)(x) \quad z \in \mathbb{C}, x \in \mathbb{R}^{d}$. With this kernel C.F. Dunkl has introduced in [5] a Fourier transform $\mathcal{F}_{D}$ called Dunkl transform. This transform has been studied in [5], [7], [19]). The main results obtained are a Plancherel theorem and Paley-Wiener theorems for functions and distributions (see [5], [7], [19]).

In this paper we consider the Silva space $\mathcal{G}$ defined as the set of functions $\varphi$ belongs to $\mathcal{E}\left(\mathbb{R}^{d}\right)$ such that for any $h, p>0$

$$
\sup _{x \in \mathbb{R}^{d}, \mu \in \mathbb{N}^{d}} \frac{e^{p\|x\|}\left|\partial^{\mu} \varphi(x)\right|}{h^{|\mu|} \mu !}<\infty .
$$


We prove that the Dunkl transform is an isomorphism from $\mathcal{G}$ onto itself. Next we extended this result to $\mathcal{G}^{\prime}$ (the dual topological space of $\mathcal{G}$ ).

The Sobolev space $W^{s, p}\left(\mathbb{R}^{d}\right)$ serves as a very useful tool in the theory of partial differential equations, which is defined as follows

$$
W^{s, p}\left(\mathbb{R}^{d}\right)=\left\{u \in \mathcal{S}^{\prime}\left(\mathbb{R}^{d}\right),\left(1+\|\xi\|^{2}\right)^{\frac{s}{p}} \mathcal{F}(u) \in L^{p}\left(\mathbb{R}^{d}\right)\right\} .
$$

In this paper we introduce the generalized Dunkl-Sobolev space of exponential type $W_{\mathcal{G}, k}^{s, p}\left(\mathbb{R}^{d}\right)$ by replacing $\left(1+\|\xi\|^{2}\right)^{\frac{s}{p}}$ by an exponential weight function defined as follow

$$
W_{\mathcal{G}, k}^{s, p}\left(\mathbb{R}^{d}\right)=\left\{u \in \mathcal{G}^{\prime}, e^{s\|\xi\|} \mathcal{F}_{D}(u) \in L_{k}^{p}\left(\mathbb{R}^{d}\right)\right\},
$$

where $L_{k}^{p}\left(\mathbb{R}^{d}\right)$ are the Lebesgue spaces associated with the Dunkl transform.

We investigate their properties such as the imbedding theorems and the structure theorems. In fact, the imbedding theorems means that for $s>0, u$ is in $W_{\mathcal{G}, k}^{s, p}\left(\mathbb{R}^{d}\right)$ can be analytically continued to the set $\left\{z \in \mathbb{C}^{d}, /|\operatorname{Im} z|<s\right\}$. For the structure theorems we prove that for $s>0 u$ is in $W_{\mathcal{G}, k}^{-s, 2}\left(\mathbb{R}^{d}\right)$ can be represented as an infinite sum of Dunkl operators of square integrable functions $g_{\mu}$, in other words,

$$
u=\sum_{\mu \in \mathbb{N}^{d}} \frac{s^{|\mu|}}{\mu !} T^{\mu} g_{\mu},
$$

where $T^{\mu}=T_{1}^{\mu_{1}} \times \ldots \times T_{d}^{\mu_{d}}, \mu=\left(\mu_{1}, \ldots, \mu_{d}\right)$. We prove also that the DunklSobolev spaces are stable by multiplication of the functions of the Silva spaces. As applications on the Dunkl-Sobolev spaces of exponential type, we study the action for the class of pseudo differential-difference operators and we apply the theory of reproducing kernels on these spaces.

We conclude this introduction with a summary of the contents of this paper. In section 2, we recall harmonic analysis associated with the Dunkl operators which we need in the sequence. We define in section 3, the DunklSobolev spaces of exponential type $W_{\mathcal{G}, k}^{s, p}\left(\mathbb{R}^{d}\right)$ and we give their properties. In section 4, we give two applications on the Dunkl-Sobolev spaces of exponential type. More precisely in the first application we introduce certain classes of symbols of exponential type whose symbols have suitable growth condition and its associated to the pseudo-differential-difference operators of exponential type. We show that these pseudo-differential-difference operators naturally act on the generalized Sobolev spaces of exponential type. Finally using the theory of reproducing kernels some applications are given for these spaces. 


\section{Harmonic analysis associated with the Dunkl operators}

In the first two subsections we collect some notations and results on Dunkl operators and the Dunkl kernel (see [3], [4]).

2.1 Reflection groups, root system and multiplicity functions. We consider $\mathbb{R}^{d}$ with the Euclidean scalar product $\langle.,$.$\rangle and \|x\|=$ $\sqrt{\langle x, x\rangle}$. On $\mathbb{C}^{d},\|\cdot\|$ denotes also the standard Hermitian norm while for all $z=\left(z_{1}, \ldots, z_{d}\right), w=\left(w_{1}, \ldots, w_{d}\right) \in \mathbb{C}^{d},\langle z, w\rangle=\sum_{j=1}^{d} z_{j} \bar{w}_{j}$. For $\alpha \in \mathbb{R}^{d} \backslash\{0\}$, let $\sigma_{\alpha}$ be the reflection in the hyperplan $H_{\alpha} \subset \mathbb{R}^{d}$ orthogonal to $\alpha$, i.e.

$$
\sigma_{\alpha}(x)=x-2 \frac{\langle\alpha, x\rangle}{\|\alpha\|^{2}} \alpha .
$$

A finite set $R \subset \mathbb{R}^{d} \backslash\{0\}$ is called a root system if $R \cap \mathbb{R} . \alpha=\{\alpha,-\alpha\}$ and $\sigma_{\alpha} R=R$ for all $\alpha \in R$. For a given root system $R$ the reflections $\sigma_{\alpha}, \alpha \in R$, generate a finite group $W \subset O(d)$, the reflection group associated with $R$. All reflections in $W$ correspond to suitable pairs of roots. For a given $\beta \in \mathbb{R}^{d} \backslash \bigcup_{\alpha \in R} H_{\alpha}$ we fix the positive subsystem $R_{+}=\{\alpha \in R /\langle\alpha, \beta\rangle>0\}$, then for each $\alpha \in R$, either $\alpha \in R_{+}$or $-\alpha \in R_{+}$. We will assume that $\langle\alpha, \alpha\rangle=2$ for all $\alpha \in R_{+}$. A function $k: R \rightarrow \mathbb{C}$ on a root system $R$ is called a multiplicity function if it is invariant under the action of the associated reflection group $W$. If one regards $k$ as a function on the corresponding reflections, this means that $k$ is constant on the conjugacy classes of reflections in $W$. For abbreviation, we introduce the index

$$
\gamma=\gamma(k)=\sum_{\alpha \in R_{+}} k(\alpha)
$$

Moreover, $\omega_{k}$ denotes the weight function

$$
\omega_{k}(x)=\prod_{\alpha \in R_{+}}|\langle\alpha, x\rangle|^{2 k(\alpha)},
$$

which is $W$-invariant and homogeneous of degree $2 \gamma$. We introduce the Mehta-type constant

$$
c_{k}=\int_{\mathbb{R}^{d}} e^{-\frac{\|x\|^{2}}{2}} \omega_{k}(x) d x .
$$


Remark. For $d=1$ and $W=\mathbf{Z}_{2}$, the multiplicity function $k$ is a single parameter denoted $\gamma>0$ and we have $\omega_{k}(x)=|x|^{2 \gamma}$ for all $x \in \mathbb{R}$.

2.2 Dunkl operators, Dunkl kernel and Dunkl intertwining operator and its dual. We use the following notations:

- $C\left(\mathbb{R}^{d}\right)\left(\right.$ resp $\left.C_{c}\left(\mathbb{R}^{d}\right)\right)$ : the space of continuous functions on $\mathbb{R}^{d}$ (resp. with compact support).

- $C^{p}\left(\mathbb{R}^{d}\right)\left(\right.$ resp $\left.C_{c}^{p}\left(\mathbb{R}^{d}\right)\right)$ : the space of functions of class $C^{p}$ on $\mathbb{R}^{d}$ (resp. with compact support).

- $\mathcal{E}\left(\mathbb{R}^{d}\right)$ : the space of $C^{\infty}$-functions on $\mathbb{R}^{d}$.

- $\mathcal{S}\left(\mathbb{R}^{d}\right)$ : the space of $C^{\infty}$-functions on $\mathbb{R}^{d}$ which are rapidly decreasing as their derivatives.

- $D\left(\mathbb{R}^{d}\right)$ : the space of $C^{\infty}$-functions on $\mathbb{R}^{d}$ which are of compact support.

- $\mathcal{E}^{\prime}\left(\mathbb{R}^{d}\right)$ : the space of distributions on $\mathbb{R}^{d}$ with compact support. It is the topological dual of $\mathcal{E}\left(\mathbb{R}^{d}\right)$.

- $\mathcal{S}^{\prime}\left(\mathbb{R}^{d}\right)$ : the space of temperate distributions on $\mathbb{R}^{d}$. It is the topological dual of $\mathcal{S}\left(\mathbb{R}^{d}\right)$.

We provide these spaces with the classical topology.

The Dunkl operators $T_{j}, j=1, \ldots, d$, on $\mathbb{R}^{d}$ associated with the finite reflection group $W$ and the multiplicity function $k$ are given by

(5) $\quad T_{j} f(x)=\frac{\partial}{\partial x_{j}} f(x)+\sum_{\alpha \in R_{+}} k(\alpha) \alpha_{j} \frac{f(x)-f\left(\sigma_{\alpha}(x)\right)}{\langle\alpha, x\rangle}, \quad f \in C^{1}\left(\mathbb{R}^{d}\right)$.

In the case $k=0$, the $T_{j}, j=1, \ldots, d$, reduce to the corresponding partial derivatives. In this paper, we will assume throughout that $k \geq 0$ and $\gamma>0$.

Dunkl Laplacian $\triangle_{k}$ on $\mathbb{R}^{d}$ is defined by

$$
\triangle_{k} f=\sum_{j=1}^{d} T_{j}^{2} f=\triangle f+2 \sum_{\alpha \in R_{+}} k_{\alpha} \delta_{\alpha}(f), \quad f \in C^{2}\left(\mathbb{R}^{d}\right),
$$

where $\triangle=\sum_{j=1}^{d} \partial_{j}^{2}$ the Laplacian on $\mathbb{R}^{d}$ and

$$
\delta_{\alpha}(f)(x)=\frac{\langle\nabla f(x), \alpha\rangle}{\langle\alpha, x\rangle}-\frac{f(x)-f\left(\sigma_{\alpha}(x)\right)}{\langle\alpha, x\rangle^{2}},
$$


with $\nabla f$ the gradient of $f$. For $y \in \mathbb{R}^{d}$, the system

$$
\left\{\begin{aligned}
T_{j} u(x, y) & =y_{j} u(x, y), & & \mathrm{j}=1, \ldots, d \\
u(0, y) & =1, & & \text { for all } y \in \mathbb{R}^{d} .
\end{aligned}\right.
$$

admits a unique analytic solution on $\mathbb{R}^{d}$, denoted by $K(x, y)$ and called Dunkl kernel. This kernel has a unique holomorphic extension to $\mathbb{C}^{d} \times \mathbb{C}^{d}$.

Example. If $d=1$ and $W=Z_{2}$, the Dunkl kernel is given by

$$
K(z, w)=j_{\gamma-\frac{1}{2}}(i z w)+\frac{z w}{2 \gamma+1} j_{\gamma+\frac{1}{2}}(i z w), \quad z, w \in \mathbb{C},
$$

where for $\alpha \geq \frac{-1}{2}, j_{\alpha}$ is the normalized Bessel function defined by

$$
j_{\alpha}(z)=2^{\alpha} \Gamma(\alpha+1) \frac{J_{\alpha}(z)}{z^{\alpha}}=\Gamma(\alpha+1) \sum_{n=0}^{\infty} \frac{(-1)^{n}\left(\frac{z}{2}\right)^{2 n}}{n ! \Gamma(\alpha+1+n)}
$$

with $J_{\alpha}$ is the Bessel function of first kind and index $\alpha$.

The Dunkl kernel possesses the following properties.

Proposition 2.1. Let $z, w \in \mathbb{C}^{d}$, and $x, y \in \mathbb{R}^{d}$.

(i) For all $\lambda \in \mathbb{C}$, we have

$$
K(z, w)=K(w, z) ; \quad K(z, 0)=1 \quad \text { and } \quad K(\lambda z, w)=K(z, \lambda w) .
$$

(ii) For all $\nu \in \mathbb{N}^{d}, x \in \mathbb{R}^{d}$ and $z \in \mathbb{C}^{d}$, we have

$$
\left|D_{z}^{\nu} K(x, z)\right| \leq\|x\|^{|\nu|} \exp (\|x\|\|\operatorname{Re} z\|)
$$

and for all $x, y \in \mathbb{R}^{d}$ :

$$
|K(i x, y)| \leq 1
$$

with $D_{z}^{\nu}=\frac{\partial^{\nu}}{\partial z_{1}^{\nu_{1}} \ldots \partial z_{d}^{\nu_{d}}}$ and $|\nu|=\nu_{1}+\ldots+\nu_{d}$.

(iii) For all $x, y \in \mathbb{R}^{d}$ and $w \in W$ we have

$$
K(-i x, y)=\overline{K(i x, y)}, \quad \text { and } \quad K(w x, w y)=K(x, y) .
$$

(iv) The function $K(x, z)$ admits for all $x \in \mathbb{R}^{d}$ and $z \in \mathbb{C}^{d}$ the following Laplace type integral representation

$$
K(x, z)=\int_{\mathbb{R}^{d}} e^{\langle y, z\rangle} d \mu_{x}(y),
$$


where $d \mu_{x}$ is a probability measure on $\mathbb{R}^{d}$, with support in the closed ball $B(0,\|x\|)$ of center 0 and radius $\|x\|$.

The Dunkl intertwining operator $V_{k}$ is defined on $C\left(\mathbb{R}^{d}\right)$ by

$$
V_{k} f(x)=\int_{\mathbb{R}^{d}} f(y) d \mu_{x}(y), \quad x \in \mathbb{R}^{d}
$$

where $d \mu_{x}$ is the measure given by the relation (13). We have

$$
K(x, z)=V_{k}\left(e^{\langle., z\rangle}\right)(x), \quad x \in \mathbb{R}^{d}, z \in \mathbb{C}^{d} .
$$

The operator ${ }^{t} V_{k}$ satisfying for $f$ in $C_{c}\left(\mathbb{R}^{d}\right)$ and $g$ in $C\left(\mathbb{R}^{d}\right)$ the relation

$$
\int_{\mathbb{R}^{d}}{ }^{t} V_{k}(f)(y) g(y) d y=\int_{\mathbb{R}^{d}} V_{k}(g)(x) f(x) \omega_{k}(x) d x,
$$

is given by

$$
{ }^{t} V_{k}(f)(y)=\int_{\mathbb{R}^{d}} f(x) d \nu_{y}(x)
$$

where $\nu_{y}$ is a positive measure on $\mathbb{R}^{d}$ with support in the set $\left\{x \in \mathbb{R}^{d}, \|\right.$ $x\|\geq\| y \|\}$. This operator is called the dual Dunkl intertwining operator.

The following theorem give some properties of the operator $V_{k}$ and ${ }^{t} V_{k}$ (see [18]).

Theorem 2.2. (i) The operator $V_{k}$ is a topological isomorphism from $\mathcal{E}\left(\mathbb{R}^{d}\right)$ onto itself satisfying the permutation relation

$$
T_{j} V_{k}(f)(x)=V_{k}\left(\frac{\partial}{\partial y_{j}} f\right)(x), \quad x \in \mathbb{R}^{d}, j=1, \ldots, d, f \in \mathcal{E}\left(\mathbb{R}^{d}\right) .
$$

(ii) The operator ${ }^{t} V_{k}$ is a topological isomorphism from $D\left(\mathbb{R}^{d}\right)$ (resp. $\mathcal{S}\left(\mathbb{R}^{d}\right)$ ) onto itself, satisfying the permutation relations

$$
{ }^{t} V_{k}\left(T_{j} f\right)(y)=\frac{\partial}{\partial y_{j}}{ }^{t} V_{k}(f)(y), y \in \mathbb{R}^{d}, j=1, \ldots, d, f \in D\left(\mathbb{R}^{d}\right) .
$$

2.3 The Dunkl transform. In this subsections we collect some notations and results on Dunkl transform (see [5],[7],[19]). We denote by $L_{k}^{p}\left(\mathbb{R}^{d}\right)$, the space of measurable functions on $\mathbb{R}^{d}$ such that 


$$
\begin{aligned}
\|f\|_{k, p} & =\left(\int_{\mathbb{R}^{d}}|f(x)|^{p} \omega_{k}(x) d x\right)^{\frac{1}{p}}<+\infty, \quad \text { if } 1 \leq p<+\infty \\
\|f\|_{k, \infty} & =\text { ess } \sup _{x \in \mathbb{R}^{d}}|f(x)|<+\infty .
\end{aligned}
$$

We shall denote by $H\left(\mathbb{C}^{d}\right)$, the space of entire functions on $\mathbb{C}^{d}$, rapidly decreasing of exponential type and by $\mathcal{H}\left(\mathbb{C}^{d}\right)$, the space of entire functions on $\mathbb{C}^{d}$, slowly increasing of exponential type. We provide these spaces with the classical topology.

The Dunkl transform of a function $f$ in $D\left(\mathbb{R}^{d}\right)$ is given by

$$
\mathcal{F}_{D}(f)(y)=\int_{\mathbb{R}^{d}} f(x) K(-i y, x) \omega_{k}(x) d x, \quad y \in \mathbb{R}^{d} .
$$

It satisfies the following properties:

(i) For $f$ in $L_{k}^{1}\left(\mathbb{R}^{d}\right)$ we have

$$
\left\|\mathcal{F}_{D}(f)\right\|_{k, \infty} \leq\|f\|_{k, 1} .
$$

(ii) For all $f$ in $\mathcal{S}\left(\mathbb{R}^{d}\right)$ we have:

$$
\mathcal{F}_{D}(f)=\mathcal{F} \circ{ }^{t} V_{k}(f),
$$

where $\mathcal{F}$ is the classical Fourier transform on $\mathbb{R}^{d}$.

(iii) For all $f$ in $\mathcal{S}\left(\mathbb{R}^{d}\right)$ we have

$$
\mathcal{F}_{D}\left(T_{j} f\right)(y)=i y_{j} \mathcal{F}_{D}(f)(y) \quad y \in \mathbb{R}^{d}, j=1, \ldots, d .
$$

(iv) For all $f$ in $L_{k}^{1}\left(\mathbb{R}^{d}\right)$ such that $\mathcal{F}_{D}(f)$ is in $L_{k}^{1}\left(\mathbb{R}^{d}\right)$, we have the inversion formula

$$
f(y)=\int_{\mathbb{R}^{d}} \mathcal{F}_{D}(f)(x) K(i x, y) \omega_{k}(x) d x, \quad \text { a.e. }
$$

Theorem 2.3. The Dunkl transform $\mathcal{F}_{D}$ is a topological isomorphism from $\mathcal{S}\left(\mathbb{R}^{d}\right)$ onto itself and from $D\left(\mathbb{R}^{d}\right)$ onto $H\left(\mathbb{C}^{d}\right)$. The inverse transform $\mathcal{F}_{D}^{-1}$ is given by

$$
\mathcal{F}_{D}^{-1}(f)(y)=\frac{c_{k}^{2}}{4^{\gamma+\frac{d}{2}}} \mathcal{F}_{D}(f)(-y), \quad y \in \mathbb{R}^{d}, f \in S\left(\mathbb{R}^{d}\right) .
$$

Theorem 2.4. The Dunkl transform $\mathcal{F}_{D}$ is a topological isomorphism from $\mathcal{S}^{\prime}\left(\mathbb{R}^{d}\right)$ onto itself and from $\mathcal{E}^{\prime}\left(\mathbb{R}^{d}\right)$ onto $\mathcal{H}\left(\mathbb{C}^{d}\right)$. 
Theorem 2.5. (i) Plancherel formula for $\mathcal{F}_{D}$ : For all $f$ in $\mathcal{S}\left(\mathbb{R}^{d}\right)$ we have

$$
\int_{\mathbb{R}^{d}}|f(x)|^{2} \omega_{k}(x) d x=\frac{c_{k}^{2}}{4^{\gamma+\frac{d}{2}}} \int_{\mathbb{R}^{d}}\left|\mathcal{F}_{D}(f)(\xi)\right|^{2} \omega_{k}(\xi) d \xi .
$$

(ii) Plancherel theorem for $\mathcal{F}_{D}$ :The renormalized Dunkl transform $f \rightarrow$ $2^{-\left(\gamma+\frac{d}{2}\right)} c_{k} \mathcal{F}_{D}(f)$ can be uniquely extended to an isometric isomorphism on $L_{k}^{2}\left(\mathbb{R}^{d}\right)$.

\subsection{The Dunkl translation operator and the Dunkl convolution} product.

Definition 2.6. Let $y \in \mathbb{R}^{d}$. The Dunkl translation operator $f \mapsto \tau_{y} f$ is defined on $\mathcal{S}\left(\mathbb{R}^{d}\right)$ by

$$
\mathcal{F}_{D}\left(\tau_{y} f\right)(x)=K(i x, y) \mathcal{F}_{D}(f)(x), \quad x \in \mathbb{R}^{d} .
$$

Example. Let $t>0$, we have

$$
\tau_{x}\left(e^{-t\|\xi\|^{2}}\right)(y)=K(2 t x, y) e^{-t\left(\|x\|^{2}+\|y\|^{2}\right)}, \quad x, y \in \mathbb{R}^{d} .
$$

Remarks. (i) For all $f$ in $L_{k}^{2}\left(\mathbb{R}^{d}\right)$ we have

$$
\left\|\tau_{x} f\right\|_{k, 2} \leq\|f\|_{k, 2} .
$$

(ii) The operator $\tau_{y}, y \in \mathbb{R}^{d}$, can also be defined on $\mathcal{E}\left(\mathbb{R}^{d}\right)$ by

$$
\tau_{y} f(x)=\left(V_{k}\right)_{x}\left(V_{k}\right)_{y}\left[\left(V_{k}\right)^{-1}(f)(x+y)\right], \quad x \in \mathbb{R}^{d} .
$$

(See [19]).

At the moment an explicit formula for the Dunkl translation operator is known only in the following two cases.

case 1: $d=1$ and $W=\mathbf{Z}_{2}$.(See [12] [17]).

case 2: For all $f \in \mathcal{E}\left(\mathbb{R}^{d}\right)$ radial we have

$$
\tau_{y} f(x)=V_{k}\left[f_{0}\left(\sqrt{\|x\|^{2}+\|y\|^{2}+2\langle x, .\rangle}\right)\right](x), \quad x, y \in \mathbb{R}^{d}
$$

with $f_{0}$ the function on $\left[0,+\infty\left[\right.\right.$ given by $f(x)=f_{0}(\|x\|)$. (See [15] [17])

Using the Dunkl translation operator, we define the Dunkl convolution product of functions as follows (See [17] [19]). 
Definition 2.7. For $f, g$ in $D\left(\mathbb{R}^{d}\right)$, we define the Dunkl convolution product by

$$
f *_{D} g(x)=\int_{\mathbb{R}^{d}} \tau_{x} f(-y) g(y) \omega(y) d y, \quad x \in \mathbb{R}^{d} .
$$

This convolution is commutative, associative and satisfies the following properties:

(i)

$$
\mathcal{F}_{D}\left(f *_{D} g\right)=\mathcal{F}_{D}(f) \mathcal{F}_{D}(g) .
$$

(ii) Let $1 \leq p, q, r \leq+\infty$, such that $\frac{1}{p}+\frac{1}{q}-\frac{1}{r}=1$. If $f$ is in $L_{k}^{p}\left(\mathbb{R}^{d}\right)$ radial and $g$ an element of $L_{k}^{q}\left(\mathbb{R}^{d}\right)$, then $f *_{D} g$ belongs to $L_{k}^{r}\left(\mathbb{R}^{d}\right)$ and we have

$$
\left\|f *_{D} g\right\|_{k, r} \leq\|f\|_{k, p}\|g\|_{k, q} .
$$

\section{The Dunkl-Sobolev spaces of exponential type}

Definition 3.1. We denote by $\mathcal{G}$ the set of all functions $\varphi$ in $\mathcal{E}\left(\mathbb{R}^{d}\right)$ such that for any $h, p>0$

$$
N_{p, h}(\varphi)=\sup _{x \in \mathbb{R}^{d}, \mu \in \mathbb{N}^{d}} \frac{e^{p\|x\|}\left|\partial^{\mu} \varphi(x)\right|}{h^{|\mu|} \mu !}<\infty .
$$

The topology in $\mathcal{G}$ is defined by the above seminorms.

Lemma 3.2. Let $\phi$ be in $\mathcal{G}$. Then for every $h, p>0$

$$
\sup _{x \in \mathbb{R}^{d}, \mu \in \mathbb{N}^{d}} \frac{e^{p\|x\|}\left|T^{\mu} \varphi(x)\right|}{h^{|\mu|} \mu !}<\infty .
$$

Proof. The result follows using ([10], Proposition 5.1) and by a simple calculation.

Theorem 3.3. The transform $\mathcal{F}_{D}$ is a topological isomorphism from $\mathcal{G}$ onto itself.

Proof. From the relations (16),(18) and Lemma 3.2 we see that ${ }^{t} V_{k}$ is continuous from $\mathcal{G}$ onto itself. On the other hand J.Chung and all in [1] has proved that the classical Fourier transform is an isomorphism from $\mathcal{G}$ onto itself. Thus from the relation (21) we deduce that $\mathcal{F}_{D}$ is continuous from $\mathcal{G}$ 
onto itself. Finally using that $\mathcal{G}$ is include in $\mathcal{S}\left(\mathbb{R}^{d}\right), \mathcal{F}_{D}$ is an isomorphism from $\mathcal{S}\left(\mathbb{R}^{d}\right)$ onto itself and the relation (24) we obtain the result.

We denote by $\mathcal{G}^{\prime}$ the strong dual of the space $\mathcal{G}$.

Definition 3.4. The Dunkl transform of a distribution $S$ in $\mathcal{G}^{\prime}$ is defined by

$$
\left\langle\mathcal{F}_{D}(S), \psi\right\rangle=\left\langle S, \mathcal{F}_{D}(\psi)\right\rangle, \psi \in \mathcal{G}
$$

The following result is immediately from Theorem 3.3.

Corollary 3.5. The transform $\mathcal{F}_{D}$ is a topological isomorphism from $\mathcal{G}^{\prime}$ onto itself.

Let $\tau$ be in $\mathcal{G}^{\prime}$. We define $T_{j} \tau, j=1, \ldots, d$, by

$$
\left\langle T_{j} \tau, \psi\right\rangle=-\left\langle\tau, T_{j} \psi\right\rangle, \quad \text { for all } \quad \psi \in \mathcal{G} \text {. }
$$

This functional satisfies the following property

$$
\mathcal{F}_{D}\left(T_{j} \tau\right)=i y_{j} \mathcal{F}_{D}(\tau), \quad j=1, \ldots, d .
$$

Definition 3.6. Let $s$ be in $\mathbb{R}, 1 \leq p<\infty$, we define the space $W_{\mathcal{G}, k}^{s, p}\left(\mathbb{R}^{d}\right)$ by

$$
\left\{u \in \mathcal{G}^{\prime}: e^{s\|\xi\|} \mathcal{F}_{D}(u) \in L_{k}^{p}\left(\mathbb{R}^{d}\right)\right\} .
$$

The norm on $W_{\mathcal{G}, k}^{s, p}\left(\mathbb{R}^{d}\right)$ is given by

$$
\|u\|_{W_{\mathcal{G}, k}^{s, p}}=\left(\frac{c_{k}^{2}}{4^{\gamma+\frac{d}{2}}} \int_{\mathbb{R}^{d}} e^{p s\|\xi\|}\left|\mathcal{F}_{D}(u)(\xi)\right|^{p} \omega_{k}(\xi) d \xi\right)^{\frac{1}{p}} .
$$

For $p=2$ we provide this space with the scalar product

$$
\langle u, v\rangle_{s, k}=\frac{c_{k}^{2}}{4^{\gamma+\frac{d}{2}}} \int_{\mathbb{R}^{d}} e^{2 s\|\xi\|} \mathcal{F}_{D}(u)(\xi) \overline{\mathcal{F}_{D}(v)(\xi)} \omega_{k}(\xi) d \xi,
$$

and the norm

$$
\|u\|_{W_{\mathcal{G}, k}^{s, 2}}^{2}=\langle u, u\rangle_{s, k}
$$

Proposition 3.7. $\quad$ (i) Let $1 \leq p<+\infty$. The space $W_{\mathcal{G}, k}^{s, p}\left(\mathbb{R}^{d}\right)$ provided with the norm $\|\cdot\|_{W_{\mathcal{G}, k}^{s, p}}$ is a Banach space.

(ii) $W_{\mathcal{G}, k}^{0,2}\left(\mathbb{R}^{d}\right)=L_{k}^{2}\left(\mathbb{R}^{d}\right)$.

(iii) Let $1 \leq p<+\infty$ and $s_{1}, s_{2}$ in $\mathbb{R}$ such that $s_{1} \geq s_{2}$ then

$$
W_{\mathcal{G}, k}^{s_{1}, p}\left(\mathbb{R}^{d}\right) \hookrightarrow W_{\mathcal{G}, k}^{s_{2}, p}\left(\mathbb{R}^{d}\right) .
$$


Proof. (i) It is clear that $L^{p}\left(\mathbb{R}^{d}, e^{p s\|\xi\|} \omega_{k}(\xi) d \xi\right)$ is complete and since $\mathcal{F}_{D}$ is an isomorphism from $\mathcal{G}^{\prime}$ onto itself, $W_{\mathcal{G}, k}^{s, p}\left(\mathbb{R}^{d}\right)$ is then an Banach space.

The results (ii) and (iii) are immediate from definition of the DunklSobolev space of exponential type.

Proposition 3.8. Let $1 \leq p<+\infty$, and $s_{1}, s, s_{2}$ be three real numbers satisfying $s_{1}<s<s_{2}$. Then, for all $\varepsilon>0$, there exists a nonnegative constant $C_{\varepsilon}$ such that for all $u$ in $W_{\mathcal{G}, k}^{s, p}\left(\mathbb{R}^{d}\right)$

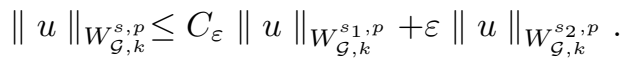

Proof. We consider $s=(1-t) s_{1}+t s_{2}$, (with $\left.t \in\right] 0,1[$ ). Moreover it is easy to see

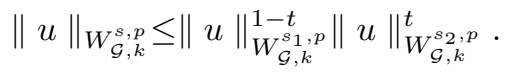

Thus

$$
\begin{aligned}
\|u\|_{W_{\mathcal{G}, k}^{s, p}} & \leq\left(\varepsilon^{-\frac{t}{1-t}}\|u\|_{W_{\mathcal{G}, k}^{s_{1}, p}}\right)^{1-t}\left(\varepsilon\|u\|_{W_{\mathcal{G}, k}^{s_{2}, p}}\right)^{t} \\
& \leq \varepsilon^{-\frac{t}{1-t}}\|u\|_{W_{\mathcal{G}, k}^{s_{1}, p}}+\varepsilon\|u\|_{W_{\mathcal{G}, k}^{s_{2}, p}} .
\end{aligned}
$$

Hence the proof is completed for $C_{\varepsilon}=\varepsilon^{-\frac{t}{1-t}}$.

Proposition 3.9. For $s$ in $\mathbb{R}, 1 \leq p<\infty$ and $\mu$ in $\mathbb{N}^{d}$, the Dunkl operator $T^{\mu}$ is continuous from $W_{\mathcal{G}, k}^{s, p}\left(\mathbb{R}^{d}\right)$ into $W_{\mathcal{G}, k}^{s-\varepsilon, p}\left(\mathbb{R}^{d}\right)$ for any $\varepsilon>0$.

Proof. Let $u$ be in $W_{\mathcal{G}, k}^{s, p}\left(\mathbb{R}^{d}\right)$, and $\mu$ in $\mathbb{N}^{d}$. From (34) we have

$$
\int_{\mathbb{R}^{d}} e^{p(s-\varepsilon)\|\xi\|}\left|\mathcal{F}_{D}\left(T^{\mu} u\right)(\xi)\right|^{p} \omega_{k}(\xi) d \xi=\int_{\mathbb{R}^{d}} e^{p(s-\varepsilon)\|\xi\|}\left\|\xi^{\mu}\right\|^{p}\left|\mathcal{F}_{D}(u)(\xi)\right|^{p} \omega_{k}(\xi) d \xi .
$$

As $\sup _{\xi \in \mathbb{R}^{d}}\|\xi\|^{|\mu|} e^{-\varepsilon\|\xi\|}<\infty$, for every $\mu \in \mathbb{N}^{d}$ and $\varepsilon>0$

$$
\int_{\mathbb{R}^{d}} e^{p(s-\varepsilon)\|\xi\|}\left|\mathcal{F}_{D}\left(T^{\mu} u\right)(\xi)\right|^{p} \omega_{k}(\xi) d \xi \leq \int_{\mathbb{R}^{d}} e^{p s\|\xi\|}\left|\mathcal{F}_{D}(u)(\xi)\right|^{p} \omega_{k}(\xi) d \xi<+\infty .
$$

Then $T^{\mu} u$ belongs to $W_{\mathcal{G}, k}^{s-\varepsilon, p}\left(\mathbb{R}^{d}\right)$, and

$$
\left\|T^{\mu} u\right\|_{W_{\mathcal{G}, k}^{s-\varepsilon, p}} \leq\|u\|_{W_{\mathcal{G}, k}^{s, p}} .
$$

Proposition 3.10. Let $P(T)=\sum_{\mu \in \mathbb{N}^{d}} a_{\mu} T^{\mu}$ be a differential-difference operator of infinite order such that there exist positive constants $C$ and $r$ 
such that

$$
\left|a_{\mu}\right| \leq C \frac{r^{|\mu|}}{\mu !} \quad \mu \in \mathbb{N}^{d}
$$

Let $1 \leq p<+\infty$ and $s$ in $\mathbb{R}$. If an element $u$ is in $W_{\mathcal{G}, k}^{s, p}\left(\mathbb{R}^{d}\right)$, then $P(T) u$ belongs to $W_{\mathcal{G}, k}^{s-r, p}\left(\mathbb{R}^{d}\right)$, and there exists a positive constant $C$ such that

$$
\|P(T) u\|_{W_{\mathcal{G}, k}^{s-r, p}} \leq C\|u\|_{W_{\mathcal{G}, k}^{s, p}} .
$$

Proof. The condition (38) gives $|P(\xi)| \leq C \exp (r\|\xi\|)$ and the assertion follows.

Proposition 3.11. Let $1 \leq p<\infty, t, s \in \mathbb{R}$. The operator $\exp \left(t \sqrt{-\triangle_{k}}\right)$ defined by

$\exp \left(t \sqrt{-\triangle_{k}}\right) u(x)=\frac{c_{k}^{2}}{4^{\gamma+\frac{d}{2}}} \int_{\mathbb{R}^{d}} K(i x, \xi) e^{t\|\xi\|} \mathcal{F}_{D}(u)(\xi) \omega_{k}(\xi) d \xi, x \in \mathbb{R}^{d}, u \in \mathcal{G}$.

is an isomorphism from $W_{\mathcal{G}, k}^{s, p}\left(\mathbb{R}^{d}\right)$ onto $W_{\mathcal{G}, k}^{s-t, p}\left(\mathbb{R}^{d}\right)$.

Proof. For $u$ in $W_{\mathcal{G}, k}^{s, p}\left(\mathbb{R}^{d}\right)$ it is easy to see that

$$
\left\|\exp \left(t \sqrt{-\triangle_{k}}\right) u\right\|_{W_{\mathcal{G}, k}^{s-t, p}}=\|u\|_{W_{\mathcal{G}, k}^{s, p}} .
$$

Then we obtain the result.

Proposition 3.12. The dual of $W_{\mathcal{G}, k}^{s, 2}\left(\mathbb{R}^{d}\right)$ can be identified with $W_{\mathcal{G}, k}^{-s, 2}\left(\mathbb{R}^{d}\right)$. The relation of the identification is given by

$$
\langle u, v\rangle_{k}=\frac{c_{k}^{2}}{4^{\gamma+\frac{d}{2}}} \int_{\mathbb{R}^{d}} \mathcal{F}_{D}(u)(\xi) \overline{\mathcal{F}_{D}(v)(\xi)} \omega_{k}(\xi) d \xi
$$

with $u \in W_{\mathcal{G}, k}^{s, 2}\left(\mathbb{R}^{d}\right)$ and $v \in W_{\mathcal{G}, k}^{-s, 2}\left(\mathbb{R}^{d}\right)$.

Proof. Let $u \in W_{\mathcal{G}, k}^{s, 2}\left(\mathbb{R}^{d}\right)$ and $v \in W_{\mathcal{G}, k}^{-s, 2}\left(\mathbb{R}^{d}\right)$. The Cauchy-Schwartz inequality give that

$$
\left|\langle u, v\rangle_{k}\right| \leq\|u\|_{W_{\mathcal{G}, k}^{s, 2}}\|v\|_{W_{\mathcal{G}, k}^{-s, 2}} .
$$

Thus for $v \in W_{\mathcal{G}, k}^{-s, 2}\left(\mathbb{R}^{d}\right)$ fixed we see that $u \longmapsto\langle u, v\rangle_{k}$ is continuous linear form on $W_{\mathcal{G}, k}^{s, 2}\left(\mathbb{R}^{d}\right)$ whose norm does net exceed $\|v\|_{W_{\mathcal{G}, k}^{-s, 2}}$.

Taking $u_{0}=\mathcal{F}_{D}^{-1}\left(e^{-2 s\|\xi\|} \mathcal{F}_{D}(v)\right)$ element of $W_{\mathcal{G}, k}^{s, 2}\left(\mathbb{R}^{d}\right)$ one can obtain 
$\left\langle u_{0}, v\right\rangle_{k}=\|v\|_{W_{\mathcal{G}, k}^{-s, 2}}$. Thus the norm of $u \longmapsto\langle u, v\rangle_{k}$ is equal to $\|v\|_{W_{\mathcal{G}, k}^{-s, 2}}$, and we have then an isometry $W_{\mathcal{G}, k}^{-s, 2}\left(\mathbb{R}^{d}\right) \longrightarrow\left(W_{\mathcal{G}, k}^{s, 2}\left(\mathbb{R}^{d}\right)\right)^{\prime}$.

Conversely, let $L \in\left(W_{\mathcal{G}, k}^{s, 2}\left(\mathbb{R}^{d}\right)\right)^{\prime}$. By the Riesz representation theorem and (35) there exists $w \in W_{\mathcal{G}, k}^{s, 2}\left(\mathbb{R}^{d}\right)$ such that for all $u \in W_{\mathcal{G}, k}^{s, 2}\left(\mathbb{R}^{d}\right)$,

$$
L(u)=\langle u, w\rangle_{s, k}=\frac{c_{k}^{2}}{4^{\gamma+\frac{d}{2}}} \int_{\mathbb{R}^{d}} e^{2 s\|\xi\|} \mathcal{F}_{D}(u)(\xi) \overline{\mathcal{F}_{D}(w)(\xi)} \omega_{k}(\xi) d \xi .
$$

If we set $v=\mathcal{F}_{D}^{-1}\left(e^{2 s\|\xi\|} \mathcal{F}_{D}(w)\right)$ then $v \in W_{\mathcal{G}, k}^{-s, 2}\left(\mathbb{R}^{d}\right)$ and $L(u)=\langle u, v\rangle_{k}$ for all $u \in W_{\mathcal{G}, k}^{s, 2}\left(\mathbb{R}^{d}\right)$, which completes the proof.

Proposition 3.13. Let $s>0$. Then every $u$ in $W_{\mathcal{G}, k}^{-s, 2}\left(\mathbb{R}^{d}\right)$ can be represented as an infinite sum of Dunkl operators of square integrable functions $g_{\mu}$, in other words,

$$
u=\sum_{\mu \in \mathbb{N}^{d}} \frac{s^{|\mu|}}{\mu !} T^{\mu} g_{\mu} .
$$

Proof. If $u$ in $W_{\mathcal{G}, k}^{-s, 2}\left(\mathbb{R}^{d}\right)$ then by definition $e^{-s\|\xi\|} \mathcal{F}_{D}(u)(\xi) \in L_{k}^{2}\left(\mathbb{R}^{d}\right)$, which by Theorem 2.5 implies that

$$
\mathcal{F}_{D}(g)(\xi)=\frac{\mathcal{F}_{D}(u)(\xi)}{\sum_{\mu \in \mathbb{N}^{d}} \frac{s^{|\mu|}}{\mu !}\left\|\xi^{\mu}\right\|} \in L_{k}^{2}\left(\mathbb{R}^{d}\right) .
$$

Hence, we have

$$
\begin{aligned}
\mathcal{F}_{D}(u) & =\sum_{\mu \in \mathbb{N}^{d}} \frac{s^{|\mu|}}{\mu !}\left\|\xi^{\mu}\right\| \mathcal{F}_{D}(g), \text { in } \mathcal{G}^{\prime} \\
& =\sum_{\mu \in \mathbb{N}^{d}} \frac{s^{|\mu|}}{\mu !}(i \xi)^{\mu}\left(\frac{\left\|\xi^{\mu}\right\|}{(i \xi)^{\mu}} \mathcal{F}_{D}(g)\right), \text { in } \mathcal{G}^{\prime} \\
& =\sum_{\mu \in \mathbb{N}^{d}} \frac{s^{|\mu|}}{\mu !}(i \xi)^{\mu} \mathcal{F}_{D}\left(g_{\mu}\right), \text { in } \mathcal{G}^{\prime} \\
& =\sum_{\mu \in \mathbb{N}^{d}} \frac{s^{|\mu|}}{\mu !} \mathcal{F}_{D}\left(T^{\mu} g_{\mu}\right) \text { in } \mathcal{G}^{\prime},
\end{aligned}
$$

where

$$
\mathcal{F}_{D}\left(g_{\mu}\right)(\xi)=\frac{\left\|\xi^{\mu}\right\|}{(i \xi)^{\mu}} \mathcal{F}_{D}(g)(\xi) \in L_{k}^{2}\left(\mathbb{R}^{d}\right)
$$

and the proof is complete. 
Proposition 3.14. Let $1 \leq p<\infty$. Then every $u \in W_{\mathcal{G}, k}^{s, p}\left(\mathbb{R}^{d}\right)$ is a holomorphic function in the strip $\left\{z \in \mathbb{C}^{d},\|\operatorname{Imz}\|<s\right\}$ for $s>0$.

Proof. Let

$$
u(z)=\frac{c_{k}^{2}}{4^{\gamma+\frac{d}{2}}} \int_{\mathbb{R}^{d}} K(i z, \xi) \mathcal{F}_{D}(u)(\xi) \omega_{k}(\xi) d \xi, z=x+i y .
$$

From (10), for each $\mu$ in $\mathbb{N}^{d}$ we have

$$
\left|D_{z}^{\mu}\left(K(i z, \xi) \mathcal{F}_{D}(u)(\xi)\right)\right| \leq\|\xi\|^{|\mu|} e^{\|y\|\|\xi\|}\left|\mathcal{F}_{D}(u)(\xi)\right| .
$$

On the other hand from Cauchy-Schwartz inequality we have

$$
\begin{aligned}
& \int_{\mathbb{R}^{d}}\left\|\xi^{\mu}\right\| e^{\|y\|\|\xi\|}\left|\mathcal{F}_{D}(u)(\xi)\right| \omega_{k}(\xi) d \xi \\
& \leq\left(\int_{\mathbb{R}^{d}}\|\xi\|^{q|\mu|} e^{q\|\xi\|(\|y\|-s)} \omega_{k}(\xi) d \xi\right)^{\frac{1}{q}}\|u\|_{W_{\mathcal{G}, k}^{s, p}}
\end{aligned}
$$

Since the integral in the last part of the above inequality is integrable if $\|y\|<s$, the result follows by the theorem of holomorphy under integral sign.

Notations. Let $m \in \mathbb{N}$. We denote by:

- $\mathcal{E}_{m}^{\prime}\left(\mathbb{R}^{d}\right)$ : the space of distributions on $\mathbb{R}^{d}$ with compact support and order less than or equal to $m$.

- $\mathcal{E}_{\text {exp }, m}^{\prime}\left(\mathbb{R}^{d}\right)$ : the space of distributions $u$ in $\mathcal{E}_{m}^{\prime}\left(\mathbb{R}^{d}\right)$ such that there exists a positive constant $C$ such that

$$
\left|\mathcal{F}_{D}(u)(\xi)\right| \leq C e^{m\|\xi\|} .
$$

Proposition 3.15. (i) Let $1 \leq p<+\infty$. For $s \in \mathbb{R}$ such that $s<-m$, we have

$$
\mathcal{E}_{\text {exp }, m}^{\prime}\left(\mathbb{R}^{d}\right) \subset W_{\mathcal{G}, k}^{s, p}\left(\mathbb{R}^{d}\right) .
$$

(ii) Let $1 \leq p<+\infty$. We have For $s<0$ and $m$ integer

$$
\mathcal{E}_{m}^{\prime}\left(\mathbb{R}^{d}\right) \subset W_{\mathcal{G}, k}^{s, p}\left(\mathbb{R}^{d}\right) .
$$

Proof. The proof uses the same idea as for ([10], Theorem 3.12) .

Theorem 3.16. Let $\psi$ be in $\mathcal{G}$. For all $s$ in $\mathbb{R}$, the mapping $u \mapsto \psi u$ from $W_{\mathcal{G}, k}^{s, 2}\left(\mathbb{R}^{d}\right)$ into itself is continuous. 
Proof. Firstly we assume that $s>0$. It is easy to see that

$$
\|v\|_{W_{\mathcal{G}, k}^{s, 2}}^{2} \leq \sum_{j=0}^{\infty} \frac{(2 s)^{j}}{j !}\|v\|_{\dot{H}_{k}^{\frac{j}{2}}\left(\mathbb{R}^{d}\right)}^{2}
$$

where $\|\cdot\|_{\dot{H}_{k}^{s}\left(\mathbb{R}^{d}\right)}$ the norm associated to homogeneous Dunkl-Sobolev space defined by

$$
\|v\|_{\dot{H}_{k}^{s}\left(\mathbb{R}^{d}\right)}^{2}=\int_{\mathbb{R}^{d}}\|\xi\|^{2 s}\left|\mathcal{F}_{D}(v)(\xi)\right|^{2} \omega_{k}(\xi) d \xi .
$$

On the other hand we proceed as Proposition 4.1 in [11] we prove that If $u, v \in \dot{H}_{k}^{s}\left(\mathbb{R}^{d}\right) \bigcap L_{k}^{\infty}\left(\mathbb{R}^{d}\right), s>0$ then $u v \in \dot{H}_{k}^{s}\left(\mathbb{R}^{d}\right)$ and

$$
\|u v\|_{\dot{H}_{k}^{s}\left(\mathbb{R}^{d}\right)} \leq C\left[\|u\|_{L_{k}^{\infty}\left(\mathbb{R}^{d}\right)}\|v\|_{\dot{H}_{k}^{s}\left(\mathbb{R}^{d}\right)}+\|v\|_{L_{k}^{\infty}\left(\mathbb{R}^{d}\right)}\|u\|_{\dot{H}_{k}^{s}\left(\mathbb{R}^{d}\right)}\right] .
$$

Thus from this we deduce that for $s>0$

$$
\begin{aligned}
\|\varphi u\|_{W_{\mathcal{G}, k}^{s, 2}}^{2} & \leq \sum_{j=0}^{\infty} \frac{(2 s)^{j}}{j !}\|\varphi u\|_{\dot{H}_{k}^{\frac{j}{2}}\left(\mathbb{R}^{d}\right)}^{2} \\
& \leq C\left[\|\varphi\|_{L_{k}^{\infty}\left(\mathbb{R}^{d}\right)}\|u\|_{W_{\mathcal{G}, k}^{s, 2}}+\|u\|_{L_{k}^{\infty}\left(\mathbb{R}^{d}\right)}\|\varphi\|_{W_{\mathcal{G}, k}^{s, 2}}\right]<+\infty .
\end{aligned}
$$

For $s=0$ the result is immediately and for $s<0$ the result is obtained by duality.

\section{Applications}

\subsection{Pseudo-differential-difference operators of exponential type.} The pseudo-differential-difference operator $A(x, T)$ associated with the symbol $a(x, \xi)$ is defined by

$$
(A(x, T) u)(x)=\frac{c_{k}^{2}}{4^{\gamma+\frac{d}{2}}} \int_{\mathbb{R}^{d}} K(i x, \xi) a(x, \xi) \mathcal{F}_{D}(u)(\xi) \omega_{k}(\xi) d \xi, u \in \mathcal{G},
$$

where $a(x, \xi)$ belongs to the class $S_{e x p, k}^{r}, r \geq 0$, defined bellow:

Definition 4.1. The function $a(x, \xi)$ is said to be in $S_{\text {exp }}^{r}$ if and only if $a(x, \xi) \in C^{\infty}\left(\mathbb{R}^{d} \times \mathbb{R}^{d}\right)$ and for each compact set $K \subset \mathbb{R}^{d}$ and each $\lambda, \mu$ in $\mathbb{N}^{d}$, there exists a constant $C_{K}=C_{\lambda, \mu, K}$ such that the estimate

$$
\left|D_{\xi}^{\lambda} D_{x}^{\mu} a(x, \xi)\right| \leq C_{K} \exp (r\|\xi\|), \quad \text { for all } \quad(x, \xi) \in K \times \mathbb{R}^{d}
$$

hold true. 
Proposition 4.2. If $a(x, \xi)$ is in $S_{\text {exp }}^{r}$ then the operator $A(x, T)$ in (40) is a well-defined mapping of $\mathcal{G}$ into $\mathcal{E}\left(\mathbb{R}^{d}\right)$.

Proof. For any compact set $K \subset \mathbb{R}^{d}$, we have

$$
|a(x, \xi)| \leq C_{K} \exp (r\|\xi\|), \text { for all }(x, \xi) \in K \times \mathbb{R}^{d} .
$$

On the other hand since $u$ is in $\mathcal{G}$ the Cauchy-Schwartz inequality give that

$$
\int_{\mathbb{R}^{d}}\left|K(i x, \xi) a(x, \xi) \mathcal{F}_{D}(u)(\xi)\right| \omega_{k}(\xi) d \xi \leq C_{K}\|u\|_{W_{\mathcal{G}, k}^{s, 2}}\left(\int_{\mathbb{R}^{d}} e^{-2(s-r)\|\xi\|_{k}} \omega_{k}(\xi) d \xi\right)^{\frac{1}{2}}
$$

is integrable for $s>r$. This prove the existence and the continuity of $(A(x, T) u)(x)$ for all $x$ in $\mathbb{R}^{d}$. Finally the result follows by using Leibniz formula.

Now we consider the symbol which belongs to the class $S_{\text {exp }}^{r, l, r a d}$ defined bellow:

Definition 4.3. Let $r, l$ in $\mathbb{R}$ be real numbers with $l>0$. The function $a(x, \xi)$ is said to be in $S_{e x p}^{r, l, r a d}$ if and only if $a(x, \xi)$ is in $C^{\infty}\left(\mathbb{R}^{d} \times \mathbb{R}^{d}\right)$, radial with respect to the first variable and for each $L>0$, and for each $\lambda, \mu$ in $\mathbb{N}^{d}$, there exists a constant $C=C_{r, l, \lambda}$ such that the estimate

$$
\left|D_{\xi}^{\lambda} D_{x}^{\mu} a(x, \xi)\right| \leq C L^{|\mu|}|\mu| \exp (r\|\xi\|-l\|x\|)
$$

hold true.

To obtain some deep and interesting results we need the following alternative from of $A(x, T)$.

Lemma 4.4. For any symbol $a(x, \xi)$ in $S_{e x p}^{r, l, r a d}$, the pseudo-differentialdifference operator $A(x, T)$ is given by:

$$
\begin{aligned}
& (A(x, T) u)(x)= \\
& \quad=\frac{c_{k}^{2}}{4^{\gamma+\frac{d}{2}}} \int_{\mathbb{R}^{d}} K(i x, \xi)\left(\int_{\mathbb{R}^{d}} \tau_{-\eta}\left(\mathcal{F}_{D}(a)(., \eta)\right)(\xi) \mathcal{F}_{D}(u)(\eta) \omega_{k}(\eta) d \eta\right) \omega_{k}(\xi) d \xi
\end{aligned}
$$

for all $u \in \mathcal{G}$ where all the involved integrals are absolutely convergent.

Proof. We proceed as [8] we see that for all $L>0$ there exist $C>0$ and $0<\tau<(L d)^{-1}$ such that

$$
\left|\mathcal{F}_{D}(a)(\xi, \eta)\right| \leq C \exp (r\|\eta\|-\tau\|\xi\|) .
$$

On the other hand since $u$ in $\mathcal{G}$, we have

$$
\left|\mathcal{F}_{D}(u)(\eta)\right| \leq C \exp (-t\|\eta\|), t>0 .
$$


Thus from the positivity for the Dunkl translation operator for the radial functions we obtain

$$
\left|\tau_{-\eta}\left(\mathcal{F}_{D}(a)(., \eta)\right)(\xi) \mathcal{F}_{D}(u)(\eta)\right| \leq C \exp (-(t-r)\|\eta\|) \tau_{-\eta}(\exp (-\tau\|\cdot\|))(\xi) .
$$

Moreover it is clear that the function

$$
\eta \mapsto \exp (-(t-r)\|\eta\|) \tau_{-\eta}(\exp (-\tau\|\cdot\|))(\xi)
$$

belongs to $L_{k}^{1}\left(\mathbb{R}^{d}\right)$ for $t>r, \tau>0$ so that

$$
\begin{aligned}
& \int_{\mathbb{R}^{d}}\left|\tau_{-\eta}\left(\mathcal{F}_{D}(a)(., \eta)\right)(\xi) \mathcal{F}_{D}(u)(\eta)\right| \omega_{k}(\eta) d \eta \\
& \leq C \int_{\mathbb{R}^{d}} \exp (-(t-r)\|\eta\|) \tau_{-\eta}(\exp (-\tau\|\cdot\|))(\xi) \omega_{k}(\eta) d \eta
\end{aligned}
$$

The right-hand side is a Dunkl convolution product of two integrable functions and hence is an integrable function on $\mathbb{R}^{d}$. Therefore the function $\xi \mapsto \int_{\mathbb{R}^{d}} \tau_{-\eta}\left(\mathcal{F}_{D}(a)(., \eta)\right)(\xi) \mathcal{F}_{D}(u)(\eta) \omega_{k}(\eta) d \eta$ is in $L_{k}^{1}\left(\mathbb{R}^{d}\right)$. Applying inverse Dunkl transform we get the result.

Now we prove the fundamental result

Theorem 4.5. Let $a \in S_{e x p}^{r, l, r a d}$ and let $A(x, T)$ be the associated pseudodifferential-difference operator. Then for all $u \in \mathcal{G}$ and all $s \in \mathbb{R}$

$$
\|A(x, T) u\|_{W_{\mathcal{G}, k}^{s, p} \leq C_{s}}\|u\|_{W_{\mathcal{G}, k}^{r+s, p}}
$$

Proof. We consider the function

$$
U_{s}(\xi)=e^{s\|\xi\|} \int_{\mathbb{R}^{d}} \tau_{-\eta}\left(\mathcal{F}_{D}(a)(., \eta)\right)(\xi) \mathcal{F}_{D}(u)(\eta) \omega_{k}(\eta) d \eta, s \in \mathbb{R} .
$$

Then invoking (41) and (29) we deduce that

$$
\begin{aligned}
\left|U_{s}(\xi)\right| \leq \int_{\mathbb{R}^{d}} & \exp ((r+s)\|\eta\|)\left|\mathcal{F}_{D}(u)(\eta)\right| \tau_{-\eta} \times \\
& \times(\exp (-(\tau-|s|)\|y\|))(\xi) \omega_{k}(\eta) d \eta, \quad s \in \mathbb{R} .
\end{aligned}
$$

The integral in (43) can be considered as a Dunkl convolution product between $f(\xi)=\exp (-(\tau-|s|) \quad\|\quad \xi\|)$ and $g(\xi)=$ $\exp ((r+s)\|\xi\|)\left|\mathcal{F}_{D}(u)(\xi)\right|$. Clearly $f$ is radial and $f \in L_{k}^{1}\left(\mathbb{R}^{d}\right)$ for $\tau>|s|$, on the other hand since $\mathcal{F}_{D}(u)$ in $\mathcal{G}$ then $g$ in $L_{k}^{p}\left(\mathbb{R}^{d}\right)$. Hence 
$f *_{D} g$ is in $L_{k}^{p}\left(\mathbb{R}^{d}\right)$ and we have

$$
\left\|f *_{D} g\right\|_{k, p} \leq\|f\|_{k, 1}\|g\|_{k, p}=C_{s}\|u\|_{W_{\mathcal{G}, k}^{r+s, p}} .
$$

Thus

$$
\|A(x, T) u\|_{W_{\mathcal{G}, k}^{s, p}}=\left\|u_{s}\right\|_{k, p} \leq\left\|f *_{D} g\right\|_{k, p} \leq C_{s}\|u\|_{W_{\mathcal{G}, k}^{r+s, p}} .
$$

This proves (42).

\subsection{The reproducing kernels.}

Proposition 4.6. For $s>0$, the Hilbert space $W_{\mathcal{G}, k}^{s, 2}\left(\mathbb{R}^{d}\right)$ admits the reproducing kernel:

$$
\Lambda_{k}(x, y)=\frac{c_{k}^{2}}{4^{\gamma+\frac{d}{2}}} \int_{\mathbb{R}^{d}} K(i x, \xi) K(-i y, \xi) e^{-2 s\|\xi\|} \omega_{k}(\xi) d \xi
$$

that is:

(i) For every $y$ in $\mathbb{R}^{d}$, the function $x \mapsto \Lambda_{k}(x, y)$ belongs to $W_{\mathcal{G}, k}^{s, 2}\left(\mathbb{R}^{d}\right)$.

(ii) For every $f$ in $W_{\mathcal{G}, k}^{s, 2}\left(\mathbb{R}^{d}\right)$ and $y$ in $\mathbb{R}^{d}$, we have

$$
\left\langle f, \Lambda_{k}(., y)\right\rangle_{s, k}=f(y) .
$$

Proof. (i) Let $y$ be in $\mathbb{R}^{d}$. From (11), the function

$$
\xi \mapsto K(-i y, \xi) e^{-2 s\|\xi\|}
$$

belongs to $L_{k}^{1}\left(\mathbb{R}^{d}\right) \cap L_{k}^{2}\left(\mathbb{R}^{d}\right)$ for $s>0$, then from Theorem 2.5 (ii) there exists a function in $L_{k}^{2}\left(\mathbb{R}^{d}\right)$, which we denote by $\Lambda_{k}(., y)$, such that

$$
\mathcal{F}_{D}\left(\Lambda_{k}(., y)\right)(\xi)=K(-i y, \xi) e^{-2 s\|\xi\|} .
$$

Thus $\Lambda_{k}(x, y)$ is given by

$$
\Lambda_{k}(x, y)=\frac{c_{k}^{2}}{4^{\gamma+\frac{d}{2}}} \int_{\mathbb{R}^{d}} K(i x, \xi) K(-i y, \xi) e^{-2 s\|\xi\|} \omega_{k}(\xi) d \xi .
$$

(ii) Let $f$ be in $W_{\mathcal{G}, k}^{s, 2}\left(\mathbb{R}^{d}\right)$ and $y$ in $\mathbb{R}^{d}$. From (35),(44) and (23) we have

$$
\left\langle f, \Lambda_{k}(., y)\right\rangle_{s, k}=\frac{c_{k}^{2}}{4^{\gamma+\frac{d}{2}}} \int_{\mathbb{R}^{d}} \mathcal{F}_{D}(f)(\xi) K(i y, \xi) \omega_{k}(\xi) d \xi=f(y) .
$$


In the follow we recall some results introduced and proved by M.Rösler in $[13]$.

Definition 4.7. The generalized heat kernel $\Gamma_{k}$ is given by

$$
\Gamma_{k}(t, x, y):=\frac{c_{k}}{(4 t)^{\gamma+\frac{d}{2}}} e^{-\frac{\|x\|^{2}+\|y\|^{2}}{4 t}} K\left(\frac{x}{\sqrt{2 t}}, \frac{y}{\sqrt{2 t}}\right) ; \quad x, y \in \mathbb{R}^{d}, t>0 .
$$

The generalized heat kernel $\Gamma_{k}$ has the following properties:

Proposition 4.8. Let $x, y$ in $\mathbb{R}^{d}$ and $t>0$. Then we have:

(i) $\Gamma_{k}(t, x, y)=\frac{c_{k}^{2}}{4^{\gamma+\frac{d}{2}}} \int_{\mathbb{R}^{d}} \exp \left(-t\|\xi\|^{2}\right) K(i x, \xi) K(-i y, \xi) \omega_{k}(\xi) d \xi$.

(ii) $\int_{\mathbb{R}^{d}} \Gamma_{k}(t, x, y) \omega_{k}(x) d x=1$.

(iii) For fixed $y$ in $\mathbb{R}^{d}$, the function $u(x, t):=\Gamma_{k}(t, x, y)$ solves the generalized heat equation:

$$
\left.\triangle_{k} u(x, t)=\frac{\partial}{\partial t} u(x, t) \text { on } \mathbb{R}^{d} \times\right] 0,+\infty[.
$$

Definition 4.9. The generalized heat semigroup $(H(t))_{t \geq 0}$ is the integral operator given for $f$ in $L_{k}^{2}\left(\mathbb{R}^{d}\right)$ by

$$
H(t) f(x):= \begin{cases}\int_{\mathbb{R}^{d}} \Gamma_{k}(t, x, y) f(y) \omega_{k}(y) d y & \text { if } \quad t>0 \\ f(x) & \text { if } t=0\end{cases}
$$

From the properties of the generalized heat kernel we have

$$
H(t) f(x):= \begin{cases}f *_{D} p_{t}(x) & \text { if } t>0 \\ f(x) & \text { if } t=0\end{cases}
$$

where

$$
p_{t}(y)=\frac{c_{k}}{(4 t)^{\gamma+\frac{d}{2}}} e^{-\frac{\|y\|^{2}}{4 t}} .
$$

Proposition 4.10. (i) Let $f$ be in $\mathcal{G}$. Then $u(x, t)=H(t) f(x)$ solves the problem

$$
\left\{\begin{array}{lll}
\left(\triangle_{k}-\frac{\partial}{\partial t}\right) u(x, t) & = & 0 \\
u(., 0) & = & f
\end{array} \text { on } \mathbb{R}^{d} \times\right] 0, \infty[
$$

(ii) The integral transform $H(t), t>0$, is a bounded linear operator from $W_{\mathcal{G}, k}^{s, 2}\left(\mathbb{R}^{d}\right)$, s in $\mathbb{R}$, into $L_{k}^{2}\left(\mathbb{R}^{d}\right)$, and we have

$$
\|H(t) f\|_{k, 2} \leq e^{\frac{s^{2}}{2 t}}\|f\|_{W_{\mathcal{G}, k}^{s, 2}}
$$


Proof. (i) This assertion follows from Definition 4.9 and Proposition 4.8 iii).

(ii) Let $f$ be in $W_{\mathcal{G}, k}^{s, 2}\left(\mathbb{R}^{d}\right)$. Using Theorem 2.5 we have

$$
\|H(t) f\|_{k, 2}^{2}=\frac{c_{k}^{2}}{4^{\gamma+\frac{d}{2}}}\left\|\mathcal{F}_{D}(H(t) f)\right\|_{k, 2}^{2} .
$$

Invoking the relations ship (31) and (45) we can write

$$
\|H(t) f\|_{k, 2}^{2}=\frac{c_{k}^{2}}{4^{\gamma+\frac{d}{2}}} \int_{\mathbb{R}^{d}} e^{-2 t\|\xi\|^{2}}\left|\mathcal{F}_{D}(f)(\xi)\right|^{2} \omega_{k}(\xi) d \xi
$$

Therefore

$$
\|H(t) f\|_{k, 2} \leq e^{\frac{s^{2}}{2 t}}\|f\|_{W_{\mathcal{G}, k}^{s, 2}}
$$

Definition 4.11. Let $r>0, t \geq 0$ and $s$ in $\mathbb{R}$. We define the Hilbert space $H_{\mathcal{G}, k}^{r, s}\left(\mathbb{R}^{d}\right)$ as the subspace of $W_{\mathcal{G}, k}^{s, 2}\left(\mathbb{R}^{d}\right)$ with the inner product:

$$
\langle f, g\rangle_{H_{\mathcal{G}, k}^{r, s}}=r\langle f, g\rangle_{s, k}+\langle H(t) f, H(t) g\rangle_{k, 2}, \quad f, g \in W_{\mathcal{G}, k}^{s, 2}\left(\mathbb{R}^{d}\right) .
$$

The norm associated to the inner product is define by:

$$
\|f\|_{H_{\mathcal{G}, k}^{r, s}}^{2}:=r\|f\|_{s, k}^{2}+\|H(t) f\|_{k, 2}^{2} .
$$

Proposition 4.12. For $s \in \mathbb{R}$, the Hilbert space $H_{\mathcal{G}, k}^{r, s}\left(\mathbb{R}^{d}\right)$ admits the following reproducing kernel:

$$
P_{r}(x, y)=\frac{c_{k}^{2}}{4^{\gamma+\frac{d}{2}}} \int_{\mathbb{R}^{d}} \frac{K(i x, \xi) K(-i y, \xi) \omega_{k}(\xi) d \xi}{r e^{2 s\|\xi\|}+e^{-2 t\|\xi\|^{2}}} .
$$

Proof. (i) Let $y$ be in $\mathbb{R}^{d}$. In the same way as in the proof of Proposition 4.6 i), we can prove that the function $x \mapsto P_{r}(x, y)$ belongs to $L_{k}^{2}\left(\mathbb{R}^{d}\right)$ and we have

$$
\mathcal{F}_{D}\left(P_{r}(., y)\right)(\xi)=\frac{K(-i y, \xi)}{r e^{2 s\|\xi\|}+e^{-2 t\|\xi\|^{2}}} .
$$

On the other hand we have

$$
\mathcal{F}_{D}\left(H(t)\left(P_{r}(., y)\right)(\xi)=\exp \left(-t\|\xi\|^{2}\right) \mathcal{F}_{D}\left(P_{r}(., y)\right)(\xi), \xi \in \mathbb{R}^{d} .\right.
$$

Hence from Theorem 2.5 (ii), we obtain 


$$
\begin{aligned}
\| H(t)\left(P_{r}(., y) \|_{k, 2}^{2}\right. & =\frac{c_{k}^{2}}{4^{\gamma+\frac{d}{2}}} \int_{\mathbb{R}^{d}} e^{-2 t\|\xi\|^{2}}\left|\mathcal{F}_{D}\left(P_{r}(., y)\right)(\xi)\right|^{2} \omega_{k}(\xi) d \xi \\
& \leq \frac{C}{r^{2}} \int_{\mathbb{R}^{d}} \frac{e^{-2 t\|\xi\|^{2}}}{e^{2 s\|\xi\|}} \omega_{k}(\xi) d \xi<\infty .
\end{aligned}
$$

Therefore we conclude that $\left\|P_{r}(., y)\right\|_{H_{\mathcal{G}, k}^{r, s}}^{2}<\infty$.

(ii) Let $f$ be in $H_{\mathcal{G}, k}^{r, s}\left(\mathbb{R}^{d}\right)$ and $y$ in $\mathbb{R}^{d}$. Then

$$
\left\langle f, P_{r}(., y)\right\rangle_{H_{\mathcal{G}, k}^{r, s}}=r I_{1}+I_{2},
$$

where

$$
I_{1}=\left\langle f, P_{r}(., y)\right\rangle_{s, k} \quad \text { and } \quad I_{2}=\left\langle H(t) f, H(t)\left(P_{r}(., y)\right)\right\rangle_{k, 2} .
$$

From (46), (35), we have

$$
I_{1}=\frac{c_{k}^{2}}{4^{\gamma+\frac{d}{2}}} \int_{\mathbb{R}^{d}} \frac{e^{2 s\|\xi\|} \mathcal{F}_{D}(f)(\xi) K(i y, \xi) \omega_{k}(\xi) d \xi}{r e^{2 s\|\xi\|}+e^{-2 t\|\xi\|^{2}}} .
$$

From (47), (46) and Theorem 2.5 ii) we have

$$
I_{2}=\frac{c_{k}^{2}}{4^{\gamma+\frac{d}{2}}} \int_{\mathbb{R}^{d}} \frac{e^{-2 t\|\xi\|^{2}} \mathcal{F}_{D}(f)(\xi) K(i y, \xi) \omega_{k}(\xi) d \xi}{r e^{2 s\|\xi\|}+e^{-2 t\|\xi\|^{2}}} .
$$

The relations (48) and (23) imply that $\left\langle f, P_{r}(., y)\right\rangle_{H_{\mathcal{G}, k}^{r, s}}=f(y)$.

4.3 Extremal function for Dunkl heat semigroup transform. In this subsection, we prove for a given function $g$ in $L_{k}^{2}\left(\mathbb{R}^{d}\right)$ that the infimum of $\left\{r\|f\|_{W_{\mathcal{G}, k}^{s, 2}}^{2}+\|g-H(t) f\|_{k, 2}^{2}, f \in W_{\mathcal{G}, k}^{s, 2}\left(\mathbb{R}^{d}\right)\right\}$ is attained at some function denoted by $f_{r, g}^{*}$, which is unique, called the extremal function. We start with the following fundamental theorem (see $[[9],[16]]$ ).

Theorem 4.13. Let $H_{K}$ be a Hilbert space admitting the reproducing kernel $K(p, q)$ on a set $E$ and $H$ a Hilbert space. Let $L: H_{K} \rightarrow H$ be a bounded linear operator on $H_{K}$ into $H$. For $r>0$, introduce the inner product in $H_{K}$ and call it $H_{K_{r}}$ as

$$
\left\langle f_{1}, f_{2}\right\rangle_{H_{K_{r}}}=r\left\langle f_{1}, f_{2}\right\rangle_{H_{K}}+\left\langle L f_{1}, L f_{2}\right\rangle_{H}
$$

Then 
(i) $H_{K_{r}}$ is the Hilbert space with the reproducing kernel $K_{r}(p, q)$ on $E$ and satisfying the equation

$$
K(., q)=\left(r I+L^{*} L\right) K_{r}(., q),
$$

where $L^{*}$ is the adjoint operator of $L: H_{K} \rightarrow H$.

(ii) For any $r>0$ and for any $g$ in $H$, the infimum

$$
\inf _{f \in H_{K}}\left\{r\|f\|_{H_{K}}^{2}+\|L f-g\|_{H}^{2}\right\}
$$

is attained by a unique function $f_{r, g}^{*}$ in $H_{K}$ and this extremal function is given by

$$
f_{r, g}^{*}(p)=\left\langle g, L K_{r}(., p)\right\rangle_{H} .
$$

We can now state the main result of this paragraph.

Theorem 4.14. Let $s \in \mathbb{R}$. For any $g$ in $L_{k}^{2}\left(\mathbb{R}^{d}\right)$ and for any $r>0$, the infimum

$$
\inf _{f \in W_{\mathcal{G}, k}^{s, 2}\left(\mathbb{R}^{d}\right)}\left\{r\|f\|_{W_{\mathcal{G}, k}^{s, 2}}^{2}+\|g-H(t) f\|_{k, 2}^{2}\right\}
$$

is attained by a unique function $f_{r, g}^{*}$ given by

$$
f_{r, g}^{*}(x)=\int_{\mathbb{R}^{d}} g(y) Q_{r}(x, y) \omega_{k}(y) d y
$$

where

$$
Q_{r}(x, y)=Q_{r, s}(x, y)=\frac{c_{k}^{2}}{4^{\gamma+\frac{d}{2}}} \int_{\mathbb{R}^{d}} \frac{e^{-t\|\xi\|^{2}} K(i x, \xi) K(-i y, \xi)}{r e^{2 s\|\xi\|}+e^{-2 t\|\xi\|^{2}}} \omega_{k}(\xi) d \xi .
$$

Proof. By Proposition 4.12 and Theorem 4.13 (ii), the infimum given by (50) is attained by a unique function $f_{r, g}^{*}$, and from (49) the extremal function $f_{r, g}^{*}$ is represented by

$$
f_{r, g}^{*}(y)=\left\langle g, H(t)\left(P_{r}(., y)\right)\right\rangle_{k, 2}, \quad y \in \mathbb{R}^{d},
$$

where $P_{r}$ is the kernel given by Proposition 4.12. On the other hand we have

$$
H(t) f(x)=\frac{c_{k}^{2}}{4^{\gamma+\frac{d}{2}}} \int_{\mathbb{R}^{d}} \exp \left(-t\|\xi\|^{2}\right) \mathcal{F}_{D}(f)(\xi) K(i x, \xi) \omega_{k}(\xi) d \xi, x \in \mathbb{R}^{d} .
$$


Hence by (46), we obtain

$$
\begin{aligned}
H(t)\left(P_{r}(., y)\right)(x) & =\frac{c_{k}^{2}}{4^{\gamma+\frac{d}{2}}} \int_{\mathbb{R}^{d}} \frac{\exp \left(-t\|\xi\|^{2}\right) K(i x, \xi) K(-i y, \xi)}{r e^{2 s\|\xi\|}+e^{-2 t\|\xi\|^{2}}} \omega_{k}(\xi) d \xi \\
& =Q_{r}(x, y) .
\end{aligned}
$$

This gives (52).

Corollary 4.15. Let $s \in \mathbb{R}, \delta>0$ and $g, g_{\delta}$ in $L_{k}^{2}\left(\mathbb{R}^{d}\right)$ such that $\left\|g-g_{\delta}\right\|_{k, 2} \leq \delta$. Then

$$
\left\|f_{r, g}^{*}-f_{r, g_{\delta}}^{*}\right\|_{W_{\mathcal{G}, k}^{s, 2}} \leq \frac{\delta}{2 \sqrt{r}}
$$

Proof. From (52) and Fubini's theorem we have

$$
\mathcal{F}_{D}\left(f_{r, g}^{*}\right)(\xi)=\frac{e^{-t\|\xi\|^{2}} \mathcal{F}_{D}(g)(\xi)}{r e^{2 s\|\xi\|}+e^{-2 t\|\xi\|^{2}}}
$$

Hence

$$
\mathcal{F}_{D}\left(f_{r, g}^{*}-f_{r, g_{\delta}}^{*}\right)(\xi)=\frac{e^{-t\|\xi\|^{2}} \mathcal{F}_{D}\left(g-g_{\delta}\right)(\xi)}{r e^{2 s\|\xi\|}+e^{-2 t\|\xi\|^{2}}} .
$$

Using the inequality $(x+y)^{2} \geq 4 x y$, we obtain

$$
e^{2 s\|\xi\|}\left|\mathcal{F}_{D}\left(f_{r, g}^{*}-f_{r, g_{\delta}}^{*}\right)(\xi)\right|^{2} \leq \frac{1}{4 r}\left|\mathcal{F}_{D}\left(g-g_{\delta}\right)(\xi)\right|^{2} .
$$

Thus and from Theorem 2.5 ii) we obtain

$$
\left\|f_{r, g}^{*}-f_{r, g_{\delta}}^{*}\right\|_{W_{\mathcal{G}, k}^{s, 2}}^{2} \leq \frac{c_{k}^{2}}{4^{\gamma+\frac{d}{2}+1} r}\left\|\mathcal{F}_{D}\left(g-g_{\delta}\right)\right\|_{k, 2}^{2} \leq \frac{1}{4 r}\left\|g-g_{\delta}\right\|_{k, 2}^{2}
$$

which gives the desired result.

Corollary 4.16. Let $s>0$. If $f$ is in $W_{\mathcal{G}, k}^{s, 2}\left(\mathbb{R}^{d}\right)$ and $g=H(t) f$. Then

$$
\left\|f_{r, g}^{*}-f\right\|_{W_{\mathcal{G}, k}^{s, 2}}^{2} \rightarrow 0 \quad \text { as } r \rightarrow 0 .
$$

Proof. From (45), (53) we have

$$
\mathcal{F}_{D}(f)(\xi)=\exp \left(t\|\xi\|^{2}\right) \mathcal{F}_{D}(g)(\xi)
$$

and

$$
\mathcal{F}_{D}\left(f_{r, g}^{*}\right)(\xi)=\frac{e^{-t\|\xi\|^{2}} \mathcal{F}_{D}(g)(\xi)}{r e^{2 s\|\xi\|}+e^{-2 t\|\xi\|^{2}}}
$$


Hence

$$
\mathcal{F}_{D}\left(f_{r, g}^{*}-f\right)(\xi)=\frac{-r e^{2 s\|\xi\|} \mathcal{F}_{D}(f)(\xi)}{r e^{2 s\|\xi\|}+e^{-2 t\|\xi\|^{2}}} .
$$

Then we obtain

$$
\left\|f_{r, g}^{*}-f\right\|_{W_{\mathcal{G}, k}^{s, 2}}^{2}=\int_{\mathbb{R}^{d}} h_{r, t, s}(\xi)\left|\mathcal{F}_{D}(f)(\xi)\right|^{2} \omega_{k}(\xi) d \xi,
$$

with

$$
h_{r, t, s}(\xi)=\frac{r^{2} e^{6 s\|\xi\|}}{\left(r e^{2 s\|\xi\|}+e^{-2 t\|\xi\|^{2}}\right)^{2}} .
$$

Since

$$
\lim _{r \rightarrow 0} h_{r, t, s}(\xi)=0
$$

and

$$
\left|h_{r, t, s}(\xi)\right| \leq e^{2 s\|\xi\|},
$$

we obtain the result from the dominated convergence theorem.

Acknowledgement. We thank the anonymous referee for his deep and helpful comments.

\section{References}

[1] J. Chung, S.Y. Chung and D. Kim, A characterization for the Fourier hyperfunctions, Publ. RIMS, Kyoto Univ, 300 (1994), 203-208.

[2] J.F. van Diejen, Confluent hypergeometric orthogonal polynomials related to the rational quantum Calagero system with harmonic confissement, Comm. Math. Phys., 188 (1997), 467-497.

[3] C. F. Dunkl, Differential-difference operators associated to reflection group, Trans. Am. Math. Soc., 311 (1989), 167-183.

[4] C.F. Dunkl, Integral kernels with reflection group invariant, Can. J. Math., 43 (1991), 1213-1227.

[5] C. F. Dunkl, Hankel transforms associated to finite reflection groups, Contemp. Math., 138 (1992), 123-138.

[6] K. Hikami, Dunkl operator formalism for quantum many-body problems associated with classical root systems, J. Phys. SoS. Japan, 65 (1996), 394-401.

[7] M.F.E. de Jeu, The Dunkl transform, Invent. Math., 113, (1993), 147162.

[8] S. Lee, Generalized Sobolev spaces of exponential type, KangweonKyungki Math., J. 8 (2000), 73-86. 
[9] T. Matsuura, S. Saitoh and D.D. Trong, Approximate and analytical inversion formulas in heat conduction on multidimensional spaces, Journal of Inverse and Ill-posed Problems, 10 (2005) 479-493.

[10] H. Mejjaoli and K. Trimèche, Hypoellipticity and hypoanalliticity associated with the Dunkl Laplacian, Integ. Transf. and Special Funct., 15 (2004), 523-548.

[11] H. Mejjaoli, Littlewood-Paley decomposition associated for the Dunkl operators and paraproduct operators, JIPAM, 9 (2008), 1-25.

[12] M. Rösler. Bessel-type signed hypergroups on $\mathbb{R}$. Pr obability Measures on Groups and related structures, XI, Proceeding, Oberwalfach, 1994. (Eds.: H. Heyer and A. Mukherjea), Word Scientific, Singafore, 1995.

[13] M. Rösler, Hermite polynomials and the heat equation for Dunkl operators, Comm. Math. Phys., 192 (1998), 519-542.

[14] M. Rösler, Positivity of Dunkl's intertwining operator, Duke. Math. J., 98 (1999), 445-463.

[15] M. Rösler, A positive radial product formulas for the Dunkl kernel, Trans. Amer. Math. Soc., 355 (2003), 2413-2438.

[16] S. Saitoh. The Weierstrass transform and an isometry in the heat equation, Applicable Analysis, 16 (1983), 1-6.

[17] S. Thangavelu and Y.Xu, Convolution operator and maximal function for Dunkl transform, J. d'Analyse Mathematique, 97 (2005), 25-56.

[18] K. Trimèche, The Dunkl intertwining operator on spaces of functions and distributions and integral representation of its dual, Integ. Transf. and Special Funct., 12 (2001), 349-374.

[19] K. Trimèche,Paley-Wiener theorems for the Dunkl transform and Dunkl translation operators, Integ. Transf. and Special Funct., 13 (2002), 1738 .

Department of Mathematics

Faculty of sciences of Tunis

Campus 1060.Tunis. Tunisia

(E-mail : hatem.mejjaoli@ipest.rnu.tn)

(Received : July 2008) 


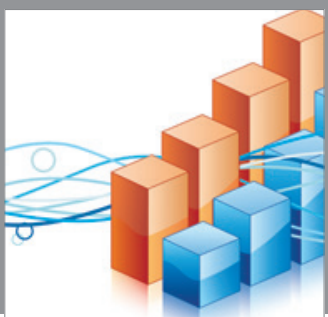

Advances in

Operations Research

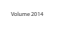

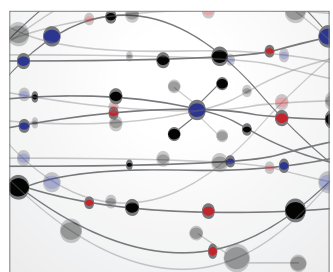

\section{The Scientific} World Journal
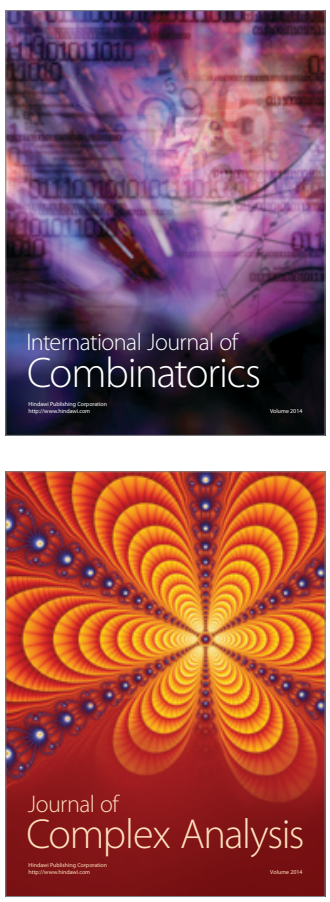

International Journal of

Mathematics and

Mathematical

Sciences
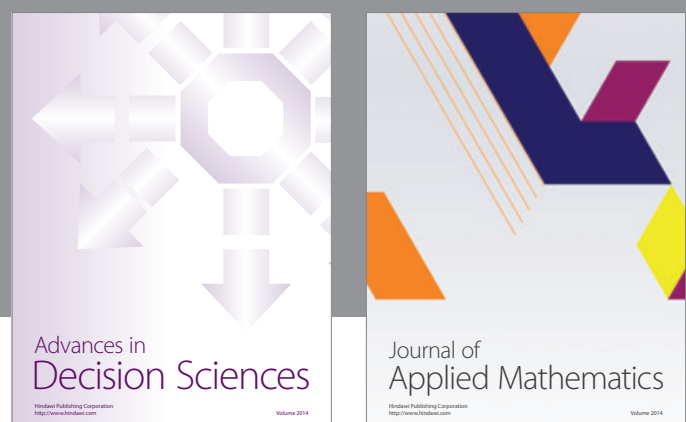

Journal of

Applied Mathematics
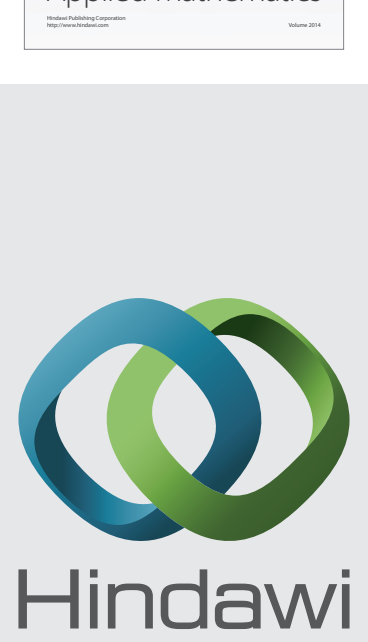

Submit your manuscripts at http://www.hindawi.com
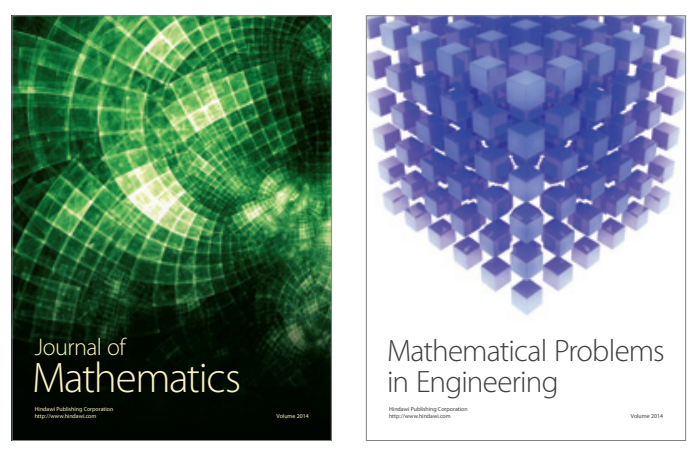

Mathematical Problems in Engineering
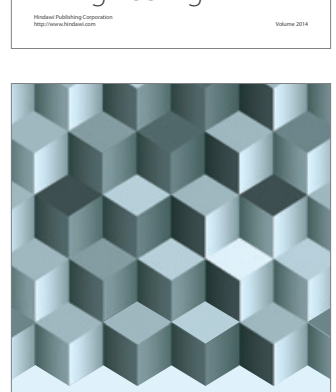

Journal of

Function Spaces
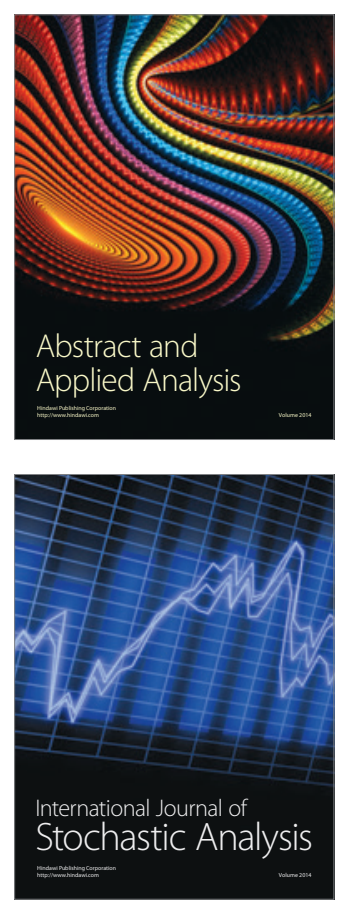

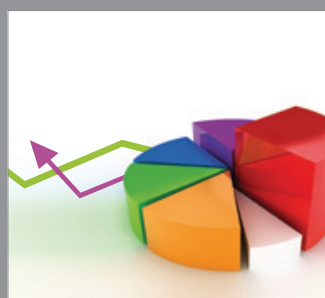

ournal of

Probability and Statistics

Promensencen
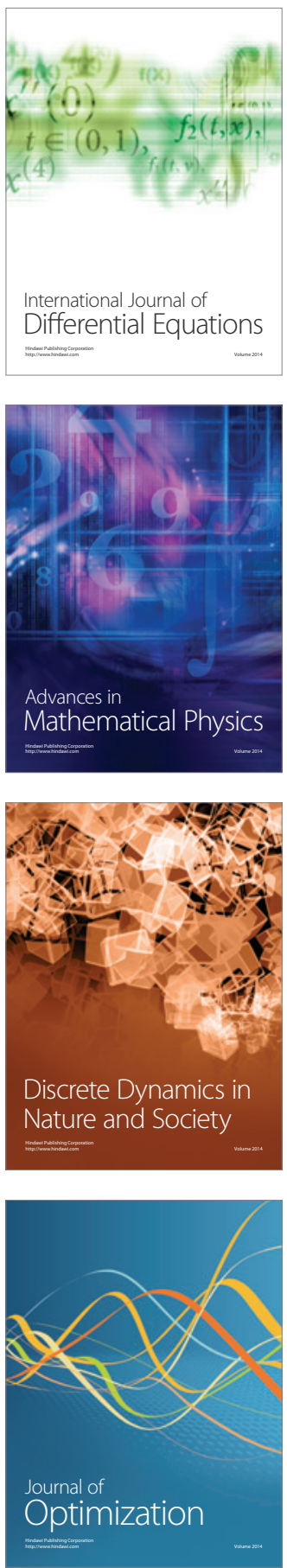